\title{
A Multiplex PCR-Based Next-Generation Sequencing Approach Has Detected a Common Large Deletion in STS Gene in a Patient with X-Linked Ichthyosis
}

\author{
Francesco Calì ${ }^{*}$, Giuseppa Maria Luana Mandarà ${ }^{2}$, Giuseppa Ruggeri ${ }^{1}$, Corrado Romano ${ }^{3}$, \\ Valeria Chiavetta ${ }^{1}$, Alda Ragalmuto ${ }^{1}$, Roberto Salluzzo' ${ }^{1}$, Valentino Romano ${ }^{4}$, \\ Marilena Galati Tardanico ${ }^{1}$, Carmelo Schepis ${ }^{5}$ \\ ${ }^{1}$ Laboratory of Molecular Genetics, Unit of Medical Genetics, IRCCS Associazione Oasi Maria SS., Troina (EN), \\ Italy \\ ${ }^{2}$ Unit of Medical Genetics, Azienda Sanitaria Provinciale di Ragusa, Ragusa, Italy \\ ${ }^{3}$ Unit of Paediatrics and Medical Genetics, IRCCS Associazione Oasi Maria SS., Troina (EN), Italy \\ ${ }^{4}$ Department of Physics and Chemistry, University of Palermo, Palermo, Italy \\ ${ }^{5}$ Unit of Dermatology, IRCCS Associazione Oasi Maria SS., Troina (EN), Italy \\ Email: "cali@oasi.en.it
}

Received 2 May 2016; accepted 12 June 2016; published 15 June 2016

Copyright (C) 2016 by authors and Scientific Research Publishing Inc.

This work is licensed under the Creative Commons Attribution International License (CC BY).

http://creativecommons.org/licenses/by/4.0/

\section{Abstract}

Several nuclear genes have been found to be linked to ichthyosis, and Next Generation Sequencing approach on panels of targeted genes has turned out to be particularly useful in analyzing diseases characterized by significant genetic and phenotypic heterogeneity. We developed a panel of 26 genes to be screened with the Ion Personal Genome Machine (PGM) for causative mutations relating to ichthyosis. Sequencing runs were obtained from a patient with ichthyosis using the Ion Torrent PGM and then processed with Ion Torrent Suite, Variant Caller, Coverage Analysis and wANNOVER tools. No causative mutations were found using Variant Caller and wANNOVER softwares, whereas the "Coverage Analysis" tool revealed a common large deletion in STS gene in a patient with X-linked ichthyosis. Identification of indels in Next Generation Sequencing (NGS) data is a veritable challenge. This study demonstrates the efficacy and effectiveness of using NGS approach to detect large deletions without resorting to specific algorithms for "indel" detection. Our results indicate that the NGS panel is a useful, rapid and cost-effective screening test for patients whose features are suggestive of a genetic etiology involving one of the genes embedded in the panel. It is an excellent alternative to Sanger sequencing as for costs, ease of analysis, and turnaround time.

\footnotetext{
${ }^{*}$ Corresponding author.
} 
Keywords

\section{X-Linked Ichthyosis, STS Gene, Next Generation Sequencing, Coverage Analysis}

\section{Introduction}

Ichthyosis is a group of clinically and etiologically heterogeneous Mendelian skin disorders, characterized by dry or scaly and thickened skin [1] [2]. A list of several genes are known to be causative of ichthyosis [3], and this list keeps getting longer and longer, as more genes are being discovered. For each gene, many mutations have been recognized. However, epidemiology has been poorly described: 13.3/10 ${ }^{6}$ liveborns in France [4], 5 $10 / 10^{5}$ liveborns in the US [5]. First recognized by Wells and Kerr [6], the X-linked ichthyosis (XLI; MIM number 308100) stems from mutations in the steroid sulfatase (STS) gene, located on Xp22.3. This X-linked recessive skin disorder is generally restricted to males. The scales are dark, nigricant, not too large, primarily over the extensor surface. By contrast, the major folds of the body appear to be spared. Palms and soles are spared by hyperlinearity. STS is involved in the metabolism of cholesterol sulfate (CSO4), needed for developing a healthy stratum corneum.

XLI is the second most common type of ichthyosis, and appears as hyperkeratosis with skin barrier dysfunction. More than $85 \%$ of patients present with either a deletion involving the entire STS gene [7]-[9], or an intragenic deletion [8]. In addition, patients may show point mutations in the STS gene [7] [10]. PCR and Sanger sequencing in a gene-by-gene approach is time-consuming and expensive. Next Generation Sequencing (NGS) [11] [12], instead, represents a major drive in many research fields, providing a powerful and efficient way to study genetic diseases. These recent technologies allow us to sequence DNA and RNA much more quickly and inexpensively than the Sanger sequencing previously used. Methods have been developed that aim to achieve analysis of genome-specific regions, by detecting all genomic variations except for CNVs, for which statistical identification approaches are still rather limited.

In our study, we used a middle-throughput targeted NGS with a panel of 26 genes, and succeeded in identifying a mutation in the STS gene in a patient showing XLI.

\section{Materials and Methods}

Patient \#3936-VR118A: Written informed consent was obtained from patient's parents. He was born after a 42-week uneventful pregnancy by Cesarean delivery due to the lack of physiologic progression. His birth weight and OFC fell within the $25^{\text {th }}-50^{\text {th }}$ centile range ( $3.450 \mathrm{kgs}$ and $35 \mathrm{cms}$, respectively), while birth length fell into the $50^{\text {th }}-75^{\text {th }}$ centile range $(52 \mathrm{cms})$. His skin was normal at birth. His weight and OFC at 3-month age (5.6 Kgs and $39.5 \mathrm{cms}$ respectively) continued to increase within the $25^{\text {th }}-50^{\text {th }}$ centile range, while his length $(60.5 \mathrm{cms})$ fell back into the $25^{\text {th }}-50^{\text {th }}$ centile range.

Clinical examination showed slight hypotonia and hyporeflexia, with good social participation, prominent forehead, deep-set eyes and nasal root, anteverted ears. His skin was widespreadly dry, with lamellar lesions mainly on the back and dorsal surface of the four limbs, with sparing of the folds. At the age of 15 months, his weight $(9.5 \mathrm{Kgs})$, length $(77 \mathrm{cms})$ and OFC $(46.2 \mathrm{cms})$ fell back into the $10^{\text {th }}-25^{\text {th }}$ centile range, versus the genetic target falling within $25^{\text {th }}-50^{\text {th }}$ centile range $(173 \mathrm{cms})$; moreover, he presented with slight delay in toddling, speech language limited to 2 - 3 bisyllabic words; his skin was diffusely dry, with patches of wide hyperkeratosis pilaris on arms, legs and back, interspersed with whitish and brownish laminar desquamating areas. At the age of about 28 months, patient's auxologic and growth parameters were all falling within the $10^{\text {th }}-25^{\text {th }}$ centile range (12 kgs, $87.5 \mathrm{cms}, 48 \mathrm{cms}$, respectively), with hyperactive behavior, utterance of several words, often not easily understandable, lack of recognition of basic colors, and no bladder and bowel control. His skin was mildly dry on the back, arms, and legs.

Patient's gDNA sample was isolated from lymphocytes, checked for degradation on agarose gel and quantified using a Qubit 2.0 Fluorometer (Invitrogen, Carlsbad, CA, USA). The primer design to create multiplex primer pools for Ion Sequencing Library was developed with Ion Ampliseq ${ }^{\mathrm{TM}}$ Designer technology (Thermo Fisher Scientific, Foster City, CA), which included 529 amplicons of 26 genes (ABCA12, ABHD5, ALOX12B, ALOXE3, AP1S1, CDSN, CSTA, CYP4F22, DSG1, DSP, FLG, GJB2, GJB3, GJB4, KRT1, KRT10, KRT2, 
LOR, NIPAL4, POMP, SLC27A4, SLURP1, SPINK5, STS, TGM1, TGM5) [3] (Oji et al., 2010). $10 \mathrm{ng}$ of gDNA were amplified using the Ion AmpliSeq ${ }^{\mathrm{TM}}$ Library Kit 2.0 in a Bio-Rad C1000 (Bio-Rad, Hercules, CA, USA), then pooled together in equimolar concentrations using the Ion Library Equalizer Kit (Thermo Fisher Scientific). Diluted library (100 pM) was then amplified through emulsion PCR using the One Touch ${ }^{\mathrm{TM}}$ Instrument (Thermo Fisher Scientific) and enriched by the One Touch ${ }^{\mathrm{TM}}$ ES Instrument (Thermo Fisher Scientific) using the Ion Personal Genome Machine (PGM) Template OT2 200 KIT, following the manufacturer's instructions. Finally, sequencing was performed on the ION PGM (Thermo Fisher Scientific) with the Ion PGM 200 Sequencing Kit (Thermo Fisher Scientific), loading samples into a 314 chip. Data from the PGM runs were processed using an Ion Torrent Suite 5.0 (Thermo Fisher Scientific), the VariantCaller 5.0 (Thermo Fisher Scientific), the wANNOVER [13] and the Ion Reporter tools (Thermo Fisher Scientific). Generated sequence files were aligned to the genomic sequence (hg19). Following the sequence alignment and extraction of Single Nucleotide Polymorphism (SNP), all the variants were filtered using Reference SNP alleles at the dbSNP138 loci (http://www.ncbi.nlm.nih.gov/SNP). Coverage analysis was performed with the Ion Torrent Coverage Analysis 5.0 (Thermo Fisher Scientific). Data were exported from the plugin and analyzed with Excel sheet (Microsoft). DNA sequences were viewed with an Integrated Genomics Viewer (IGV) [14] [15] and, finally, the disease-causing variants were analyzed and confirmed by MLPA (SALSA MLPA probemix P160STS; MRC Holland, Amsterdam, the Nethetlands).

Table 1. Amplicon coverage summary results obtained from Coverage Analysis tool.

\begin{tabular}{|c|c|c|c|}
\hline Chr & Gene & Number of amplicons & Mean of total reads \\
\hline 1 & FLG & 69 & 556 \\
\hline 1 & GJB3 & 5 & 178 \\
\hline 1 & GJB4 & 5 & 145 \\
\hline 1 & LOR & 4 & 84 \\
\hline 2 & ABCA12 & 74 & 101 \\
\hline 3 & ABHD5 & 9 & 73 \\
\hline 3 & CSTA & 3 & 93 \\
\hline 5 & NIPAL4 & 11 & 203 \\
\hline 5 & SPINK5 & 37 & 91 \\
\hline 6 & CDSN & 12 & 202 \\
\hline 6 & DSP & 60 & 112 \\
\hline 7 & AP1S1 & 7 & 75 \\
\hline 8 & SLURP1 & 3 & 325 \\
\hline 9 & SLC27A4 & 19 & 137 \\
\hline 12 & KRT1 & 17 & 144 \\
\hline 12 & KRT2 & 15 & 180 \\
\hline 13 & GJB2 & 5 & 196 \\
\hline 13 & POMP & 8 & 113 \\
\hline 14 & TGM1 & 24 & 201 \\
\hline 15 & TGM5 & 20 & 220 \\
\hline 17 & ALOX12B & 25 & 175 \\
\hline 17 & ALOXE3 & 24 & 148 \\
\hline 17 & KRT10 & 14 & 92 \\
\hline 18 & DSG1 & 26 & 67 \\
\hline 19 & CYP4F22 & 18 & 200 \\
\hline $\mathrm{X}$ & STS & 15 & 0 \\
\hline
\end{tabular}




\section{Results and Discussion}

We used the 26-gene panel, as developed in our laboratory, to genotype a patient who had met diagnostic criteria for ichthyosis. VariantCaller and wANNOVER/Ion Reporter tools analyses showed no causative mutations. Indeed, NGS technology, as known, enables the identification of missense, nonsense, splice-site as well as small insertions or deletions. Identification of indels in NGS data is a challenge, because indels are commonly analyzed using specific algorithms (Pindel, Genome Analysis Tool Kit's Unified Genotyper, HaplotypeCaller etc) [16]. In our patient, however, data from the PGM runs processed with Coverage Analysis 5.0 tool showed an alignment of reads in all panel genes, leading to a mean coverage of $186 \mathrm{X}$ across reads, except for the X-linked STS gene. This gene showed no PCR amplification (mean of total reads $=0$, see Table 1 ) in any of the exons (15 amplicons), which is compatible with a hemizygous deletion of the whole STS gene. This deletion was further confirmed by MLPA and viewed in IGV tool.

Two different methodological approaches are usually required for the detection of point mutations and large deletions in XLI: sequencing analysis and array-CGH/MLPA respectively. Here, we show that our approach enables to detect both large deletions and point mutations. Indeed, complete/partial deletions of any X-linked gene can be easily detected, in hemizygous males, by the absence of target gene PCR-amplification (STS gene) and the presence of PCR-amplification of reference housekeeping genes (other genes within the panel; see Table 1). This method is particularly useful in patients negative for deletions ( 15\%) [7]-[9], for whom point mutation retesting analysis would be otherwise requested.

Further advantages of middle-throughput targeted NGS (multiple-gene panels versus gene-by-gene approach) include significant reduction in DNA sequencing costs (less expensive than Sanger approach) [17], enhanced efficiency of genetic testing, sparing of time, number of visits, and number of tests to be performed.

\section{Conclusion}

We used a middle-throughput targeted NGS with a panel of 26 genes, and succeeded in identifying a mutation in the STS gene in a patient showing XLI. When using ION torrent (Thermo Fisher Scientific) technology, it is strongly recommended to supervise the analysis of mutations in X-linked genes through the Coverage Analysis tool (Thermo Fisher Scientific), because large deletions in males might escape current identification if analyzed through wANNOVAR and/or IonReporter exclusively.

\section{Acknowledgements}

This research study was funded by the Italian Ministry of Health. We thank Mrs. Rosi Di Giorgio for her editorial support.

\section{References}

[1] Williams, M.L. and Elias, P.M. (1986) Ichthyosis: Genetic Heterogeneity, Genodermatoses, and Genetic Counseling. Archives of Dermatology, 122, 529-531. http://dx.doi.org/10.1001/archderm.1986.01660170059019

[2] Traupe, H. (1989) The Ichthyoses: A Guide to Clinical Diagnosis, Genetic Counseling, and Therapy. Springer Verlag, New York. http://dx.doi.org/10.1007/978-3-642-73650-6

[3] Oji, V., Tadini, G., Akiyama, M., Blanchet Bardon, C., Bodemer, C., Bourrat, E., Coudiere, P., DiGiovanna, J.J., et al. (2010) Revised Nomenclature and Classification of Inherited Ichthyoses: Results of the First Ichthyosis Consensus Conference in Sorèze 2009. Journal of the American Academy of Dermatology, 63, 607-641. http://dx.doi.org/10.1016/j.jaad.2009.11.020

[4] Dreyfus, I., Chouquet, C., Ezzedine, K., Henner, S., Chiavérini, C., Maza, A., Pascal, S., Rodriguez, L., Vabres, P., Martin, L., Mallet, S., Barbarot, S., Dupuis, J. and Mazereeuw-Hautier, J. (2014) Prevalence of Inherited Ichthyosis in France: A Study Using Capture-Recapture Method. Orphanet Journal of Rare Diseases, 9, 1. http://dx.doi.org/10.1186/1750-1172-9-1

[5] Milstone, L.M., Miller, K., Haberman, M. and Dickens, J. (2012) Incidence of Moderate to Severe Ichthyosis in the United States. Archives of Dermatology, 148, 1080-1081. http://dx.doi.org/10.1001/archdermatol.2012.1702

[6] Wells, R.S. and Kerr, C.B. (1965) Genetic Classification of Ichthyosis. Archives of Dermatology, 92, 1-6. http://dx.doi.org/10.1001/archderm.1965.01600130007001

[7] Basler, E., Grompe, M., Parenti, G., Yates, J. and Ballabio, A. (1992) Identification of Point Mutations in the Steroid Sulfatase Gene of Three Patients with X-Linked Ichthyosis. The American Journal of Human Genetics, 50, 483-491. 
[8] Shapiro, L.J., Yen, P., Pomerantz, D., Martin, E., Rolewic, L. and Mohandas, T. (1989) Molecular Studies of Deletions at the Human Steroid Sulfatase Locus. Proceedings of the National Academy of Sciences of the United States of America, 86, 8477-8481. http://dx.doi.org/10.1073/pnas.86.21.8477

[9] Ballabio, A., Parenti, G., Carrozzo, R., Sebastio, G., Andria, G., Buckle, V., Fraser, N., Craig, I., Rocchi, M., Romeo, G., Jobsis, A.C. and Persico, M.G. (1987) Isolation and Characterization of a Steroid Sulfatase cDNA Clone: Genomic Deletions in Patients with X-Chromosome-Linked Ichthyosis. Proceedings of the National Academy of Sciences of the United States of America, 84, 4519-4523. http://dx.doi.org/10.1073/pnas.84.13.4519

[10] Alperin, E.S. and Shapiro, L.J. (1997) Characterization of Point Mutations with X-Linked Ichthyosis: Effects on the Structure and Function of the Steroid Sulfatase Protein. The Journal of Biological Chemistry, 272, 20756-20763. http://dx.doi.org/10.1074/jbc.272.33.20756

[11] Metzker, M.L. (2010) Sequencing Technologies—The Next Generation. Nature Reviews Genetics, 11, 31-46. http://dx.doi.org/10.1038/nrg2626

[12] Mardis, E.R. (2008) Next-Generation DNA Sequencing Methods. Annual Review of Genomics and Human Genetics, 9 , 387-402. http://dx.doi.org/10.1146/annurev.genom.9.081307.164359

[13] Yang, H. and Wang, K. (2015) Genomic Variant Annotation and Prioritization with ANNOVAR and wANNOVAR. Nature Protocols, 10, 1556-1566. http://dx.doi.org/10.1038/nprot.2015.105

[14] Robinson, J.T., Thorvaldsdóttir, H., Winckler, W., Guttman, M., Lander, E.S., Getz, G. and Mesirov, J.P. (2011) Integrative Genomics Viewer. Nature Biotechnology, 29, 24-26. http://dx.doi.org/10.1038/nbt.1754

[15] Thorvaldsdóttir, H., Robinson, J.T. and Mesirov, J.P. (2013) Integrative Genomics Viewer (IGV): High-Performance Genomics Data Visualization and Exploration. Briefings in Bioinformatics, 14, 178-192. http://dx.doi.org/10.1093/bib/bbs017

[16] Ghoneim, D.H., Myers, J.R., Tuttle, E. and Paciorkowski, A.R. (2014) Comparison of Insertion/Deletion Calling Algorithms on Human Next-Generation Sequencing Data. BMC Research Notes, 7, 864. http://dx.doi.org/10.1186/1756-0500-7-864

[17] Pareek, C.S., Smoczynski, R. and Tretyn, A. (2011) Sequencing Technologies and Genome Sequencing. Journal of Applied Genetics, 52, 413-435. http://dx.doi.org/10.1007/s13353-011-0057-x 